\title{
Article
}

\section{New Perspectives in the Development of the Artificial Sport Trainer}

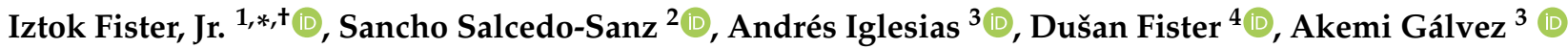 \\ and Iztok Fister 1,3 \\ 1 Faculty of Electrical Engineering and Computer Science, University of Maribor, 2000 Maribor, Slovenia; \\ iztok.fister@um.si \\ 2 Department of Signal Processing and Communications, Universidad de Alcalá, \\ 28805 Alcalá de Henares, Spain; sancho.salcedo@uah.es \\ 3 Department of Applied Mathematics and Computational Sciences, University of Cantabria, \\ 39005 Santander, Spain; iglesias@unican.es (A.I.); galveza@unican.es (A.G.) \\ 4 Faculty of Economics and Business, Razlagova 14, 2000 Maribor, Slovenia; dusan.fister@um.si \\ * Correspondence: iztok.fister1@um.si
}

Citation: Fister, I., Jr.; Salcedo-Sanz, S.; Iglesias, A.; Fister, D.; Gálvez, A.; Fister, I. New Perspectives in the Development of Artificial Sport Trainer. Appl. Sci. 2021, 11, 11452. https://doi.org/10.3390/ app112311452

Academic Editor: Jesús García Pallarés

Received: 15 November 2021 Accepted: 28 November 2021 Published: 3 December 2021

Publisher's Note: MDPI stays neutral with regard to jurisdictional claims in published maps and institutional affiliations.

Copyright: (c) 2021 by the authors. Licensee MDPI, Basel, Switzerland. This article is an open access article distributed under the terms and conditions of the Creative Commons Attribution (CC BY) license (https:// creativecommons.org/licenses/by/ $4.0 /)$.

\begin{abstract}
The rapid development of computer science and telecommunications has brought new ways and practices to sport training. The artificial sport trainer, founded on computational intelligence algorithms, has gained momentum in the last years. However, artificial sport trainer usually suffers from a lack of automatisation in realization and control phases of the training. In this study, the Digital Twin is proposed as a framework for helping athletes, during realization of training sessions, to make the proper decisions in situations they encounter. The digital twin for artificial sport trainer is based on the cognitive model of humans. This concept has been applied to cycling, where a version of the system on a Raspberry Pi already exists. The results of porting the digital twin on the mentioned platform shows promising potential for its extension to other sport disciplines.
\end{abstract}

Keywords: artificial sport trainer; digital twin; cognitive models; computational intelligence

\section{Introduction}

We could speculate that, currently, sport is top marginal thing in the world. Despite the Corona pandemic crisis, the market value of some prominent footballers is much higher than could be considered normal. Moreover, sport may be an opiate of the people. In Roman times, it served aristocrats to discipline people under slogan of "bread and circuses" by offering them an entertainment. According to linguist and philosopher Noam Chomsky [1], sport is applied by capitalism as a tool for entertaining and disciplining the working class. A lot of politicians take advantage of various visiting world-championships to scoring political points.

There are two possible directions in the further development of sport: On one hand, a professional sport strives for the best results of athletes, in individual or team sport disciplines, by hiding the training required from the full view of arbitrary observers. On the other hand, amateur sport is more open for various ideas about sport training. Frequently, the amateur athletes exchange their achievements in sport training among each other, and even share them publicly on the web in order to compare their achievement with those of other potential competitors. In cycling, virtual competitions have become very attractive, in which the professional and amateur cyclists compete virtually by overcoming some regions or parts of the cycling course as fast as possible, and their achievements, measured by wearable mobile devices, are shared publicly on the web. The result of the virtual race is the ranking of the best achievements, which is a target of new attacks of the competitors.

While professional athletes train under the watchful eye of the sport trainers, the amateur athletes are usually left to themselves. However, huge progress in modern technology, especially in computer science and telecommunication, enables automatisation of all phases 
in almost all individual as well as professional sport disciplines. In computer science, the problem has, typically, been solved using two methodologies: Machine Learning (ML) [2] and Nature Inspired (NI) [3] algorithms. Both methodologies are united under the umbrella of Computational Intelligence (CI) [4].

Araujo et al. [5] applied the ML methods for analyzing sport performance, either in team sports like football, or individual sports, like golf and climbing. Silaccio et al. [6] proposed a virtual coach for planning the road cycling training sessions based on ML methods. A virtual coach, based on similar methods, was also proposed by Rauter et al. [7], this time for planning mountain bike training sessions. Moreover, Bezobrazov et al. [8] used Artificial Neural Networks (ANN) for gesture detection and recognition in tennis. ANNs were also used in the automatisation of measuring the speed of the punches of boxers during shadow boxing using inertial measurement units [9]. A very interesting approach was tackled by the authors Chen et al. [10], where LSTM networks and a bio-inspired algorithm framework were combined, in order to recognize the action of an athlete and help them improve sports skills. Sirico et al. [11] proposed video observation and motor imagery for monitoring training loads, while Ardigo et al. [12] preferred the SuperOp device for similar purposes. All those tools can be useful for injury prevention, such as tendinopathies [13] or muscle injuries [14].

On the other hand, the NI algorithms gained a stronger momentum in the sport arena with the issuing of the paper "Planning the sport training sessions with the bat algorithm" by Fister et al. [15], and the discussion of CI in sport by Fister et al. [16], where the challenges and opportunities of this research domain are reviewed. A monograph by Fister et al. [17] presented a more complete view on the sport training area. In that work, the authors proposed the Artificial Sport Trainer (AST), whose decisions are made by CI algorithms. Potential readers are invited to look at the survey of Bonidia et al. [18] in order to discover more issues in this domain. On the other hand, a very comprehensive review on different types of fitness trackers and fitness applications was presented in [19]. The review examined the AI algorithms that are used in the smart fitness domain. A very recent study explored the wearable sensor technology and provides the use cases of ML algorithms in the field of sensor fusion [20].

This paper reveals new perspectives on the development of an AST. Indeed, the main weaknesses of the AST are automatisation of all four phases of the sport training, i.e., planning, realization, control, and evaluation [17]. While the planning and evaluation can be performed automatically, the realization and monitoring were harder to automatize at the time when the AST was issued, due to a lack of the appropriate technology. However, the rapid technology breakthrough, especially in computer science and telecommunications, has also enabled further progress of the AST. The advanced mobile sport trackers and mobile devices facilitate collecting the data acquired from remote sensors anytime and anywhere. Sensors have become ubiquitous, while the Internet of Things (IoT) allows them to be connected online on the Internet. This means that sport trainers can monitor their trainees online using suitable web applications.

The advanced technologies provide new possibilities for the AST. For an athlete during a sport training session, it does not appear only as a strong observer and critic of the athlete's performed work, but also as an adviser by making decisions relating to the current performances. Actually, the AST in this scenario can be seen as an athlete's Digital Twin (DT), which helps the athlete make decisions referring to sport training based on the same information acquired for the sensors available to both, i.e., the athlete and his/her DT. Thus, the AST is not focused on one athlete at the same time. On the contrary, it is capable of handling more athletes in parallel according to their personal characteristics. In line with this, each athlete is advised by their own DT.

The DT is modeled in such a way that it highlights the athlete's psycho-physical characteristics. At this point, we were confronted with the question of how to select the model that could be able to satisfy these demands. Consequently, we have leaned on the foundations of cognitive psychology that simulates processing of the signals from the real 
environment, makes decisions accordingly, and reacts by an action similar to what the human does [21]. The results of the DT's decision-making process is a predicted behavior, which the athlete is advised of during the sport training online. The predicted behavior can be the result of different ML methods, depending on the discipline in question.

The proposed DT can be applied to various sports disciplines, but needs the real platform for running. In an illustrative example, it was applied to cycling sport training, where the platform on Raspberry Pi was already developed by Fister et al. [22]. In porting the theoretical model of a human to the model of the real athlete in cycling, more issues have arisen, like identification of the specifics in definite sports discipline, definition of the DT for this discipline, and integration of the DT model in a specific platform (i.e., a wearable device). Due to the already existing platform, the last phase was relatively easier to implement.

The structure of the remainder of the paper is as follows: Section 2 discusses information about the applied materials and proposed method, and it is necessary for understanding the subjects that follow. In Section 3, the proposed DT is ported to a real athlete in cycling. The challenges and opportunities, as well as weaknesses, of the proposed method are discussed in Section 4. The paper concludes with Section 5, where the performed work is summarized and potential directions for future work are outlined.

\section{Materials and Methods}

The purpose of the methods section is implied to introduce those materials and methods that are necessary for the development of a DT, which serves as an adviser to an athlete during the realization of the sport training sessions. Although the proposed methodology is presented primarily for individual sport disciplines, it can also be applied for team sports.

The section consists of the following subjects:

- the basics sport training,

- the basics of a DT,

- the cognitive model of a human,

- the DT of an athlete.

In the remainder of the paper, the subjects are illustrated in detail.

\subsection{Basics of Sport Training}

The goal of sport training is developing the mental and physical performance of an athlete's body. The mental performance refers to the motivation and attitude of the athlete to sport training as a whole, while the physical performance on the influence of particular workouts they have to specific physiological categories of an athlete's body, like heart, lungs, blood, and muscles. The benefit of sport training is reflected in the athlete's fitness, and determines his/her race readiness, i.e., how competitive an athlete can be in the real competition.

According to a theory of sport [23], a training process follows four principles:

- progressive overload,

- $\quad$ specificity,

- reversibility,

- individuality.

The first principle asserts that only increasing the physical stress (i.e., overload) is a key for improving an athlete's fitness. Obviously, a human body responds to the stress either positively or negatively. In the first case, the human body reacts to the overload with fatigue, while, in the second, with overtraining that, typically, leads to injuries. The fatigue can be defeated by resting, while injuries demand recovery and, usually, also the help of medical staff. The specificity refers to effects caused by a particular workout on the particular physiological categories of an athlete's body. In line with this, two categories are distinguished: central and peripheral. The former pertains to improving fitness of the 
heart, lungs, and blood, while the latter to muscles. The principle of reversibility indicates that the fitness has been decreasing with the inactivity of the athlete. No training, no fitness improving. Also, reduced training results in losing fitness. The last principle says that each athlete is unique, and therefore must be treated by the sport trainer as an individual, and each individual needs different treatment to achieve the same level of fitness as the other.

Athletes train according to training plans prepared by either sport trainers manually, or the computer programs automatically. The training plans depend on three so-called training load indicators: duration, intensity, and frequency. Duration specifies how long an athlete must maintain the intensity at high level in overcoming the particular workouts. Indeed, intensity decreases with increasing the duration. The frequency refers to the issue of how many times the specific workout must be repeated in a specific time period.

Sport training consists of four phases: planning, realization, control, and evaluation [17]. In the planning phase, workouts are prescribed for athletes in training such that their sequence ensures them the optimal race readiness at the training period by simultaneous development of all physiological categories. The realization phase is dedicated for implementing the sport training plan on a daily basis. The quality of the performed sport training is controlled by measuring the achievement of the athlete during the workout. The measuring was conducted manually by sport trainers in the past, using, for instance, sport watches for measuring time, tapes for measuring distance, and the amount lifted during weight lifting. Recently, modern wearable devices and sport trackers have helped sport trainers by monitoring the results achieved during sport training automatically. The evaluation phase is, typically, performed after finishing some sport training period (e.g., a season). In this phase, an analysis is performed of the results achieved in competitions, and modifications in training are outlined to improve the race readiness of the athlete in the next period.

The AST [17] has performed all four phases sequentially. However, the advances of technology, especially IoT, allows for new perspectives in sport training. On the one hand, new sensors have been emerging that enable sport trainers to monitor their athletes according to new training load indicators, while, on the other hand, the existing sensors have become smaller and more accurate. These advances brought a revolution in sport training, especially in individual sport disciplines like running and cycling, where mobile devices more or less supersede the presence of the real sport trainers. Consequently, the realization and control phases started to interlace and coalesce into one unit that allows us to cover this unit using a mobile device autonomously.

The proposed new AST+ is capable of handling more athletes in parallel (Figure 1).

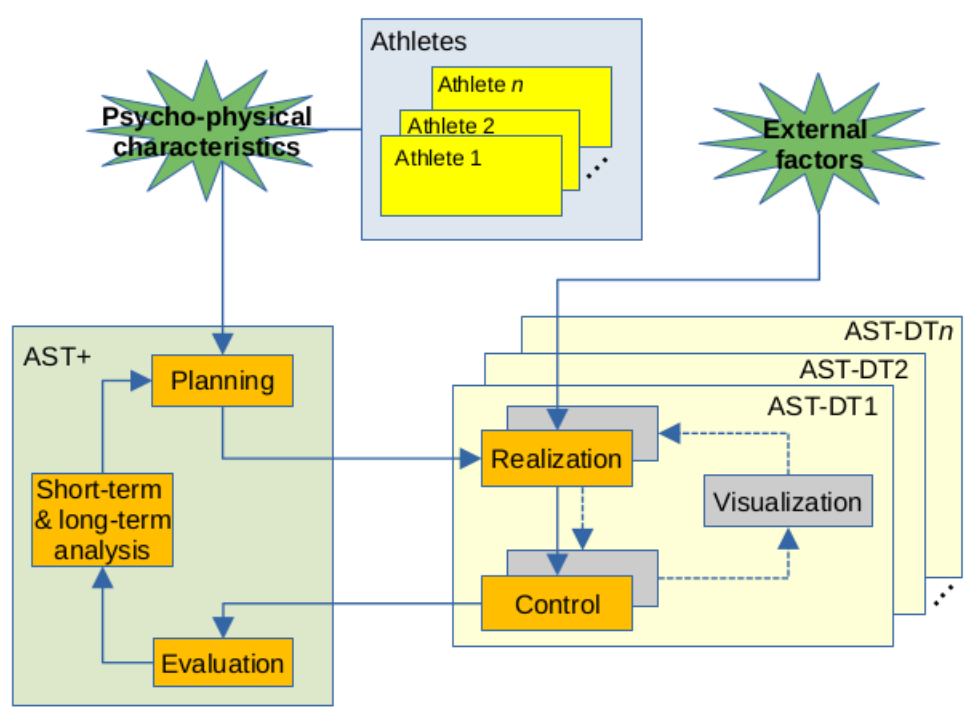

Figure 1. Concept of an advanced Artificial Sport Trainer (AST+). 
As can be seen from the figure, each athlete has their own psycho-physical characteristics (e.g., motivation, sex, age, weight) that define his/her program context. Interestingly, only two phases of the original AST have remained in AST+, i.e., planning and evaluation, while the other two, i.e., realization and control, are performed autonomously by one mobile device per athlete. Thus, each mobile device plays the role of AST+ assistant (denoted as AST-DT $i$ for $i=1$ to $n$ ), delegated for performing the realization phase of the athlete as fairly as possible, and monitoring the achieved results as reliably as possible. Obviously, the assistant operates under the full control of the AST+ in the sense that the training plan is transferred from the AST to the assistant AST-DT, while the achieved results are returned for detailed analysis in the reverse direction.

However, the assistant AST-DT incorporates a model of a particular athlete in the form of a DT. The technology is discussed in the remainder of the paper in detail.

\subsection{Basics of a Digital Twin}

A DT represents a digital replica of a physical entity, capable of understanding, analyzing, and optimizing its physical counterpart [24]. The DT maps a physical world, in which the entity is positioned, to a digital world, in which decisions are made by analyzing the real world using Artificial Intelligence (AI), Machine Learning (ML), procedures, and functions. Thus, the DT needs a model of simulated replica for the decision-making, obtains the state of the real environments by various sensors, and selects the most appropriate actions, in which physical entity affects this environment. Typically, the model consists of simulations and visualization, where the DT can predict the most appropriate action in a specific situation. Indeed, this can verify many situations in the simulated environment, and decide the best response to the particular situation without any unnecessary damage of the physical entity.

Actually, the DT framework consists of the following five elements (Figure 2):

- $\quad$ sensors

- data

- integration

- model

- actuators

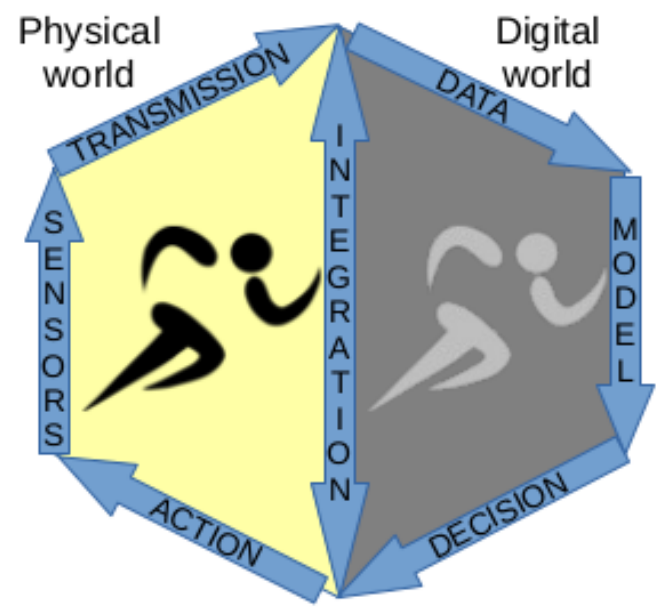

Figure 2. Concept of the digital twin (The runner's logo was taken from [25]).

Sensors enable the DT to sense a real world. We are confronted with the emergence of a huge amount of various IoT sensors [26], from which information can be extracted, even in places where this could not have been imagined in past years (e.g., power meters in cycling). Data from sensors, representing the whole image of the physical environment, are aggregated with the enterprise data by the DT. The aggregation integrates the physical entity with the digital world into a whole. The model serves for making-decisions based 
on aggregated data, while the decisions are transmitted to the physical entities. Typically, the model consists of analytical tools founded on algorithm simulations and visualization procedures. Actuators help the physical entity change the state of the physical environment by executing actions proposed by the DT. Indeed, the action is executed by human intervention manually. This means that the human must comply with the advice proposed by the DT.

\subsection{Cognitive Model of Human}

The aim of cognitive psychology is to understand the internal mentioned processes occurring during the cognition of changes in the environment, and responding to them with an appropriate action, selected after deciding on it [21]. The mentioned processes are: attention, perception, learning, memory, language, decision-making, problem solving, reasoning, and thinking. Understanding the human mind and reproducing its intelligent outcomes was the eternal desire of Artificial Intelligence [27]. Cognition psychology, and more precisely its branch of "computational cognitive science", strives to build a model capable of mimicking human cognitive functions by computer simulations [28].

In line with this, three types of models have been developed: connectionist (also neural network models), production (proposed by if-then rules), and Adaptive Control of Thought-Rational (ACT-R). While the first two kinds of models provide the fixed structure of the mind [29], the third assumes that the cognitive system consists of several modules, and the behavior is a product of interconnections among these modules. Recently, Laird et al. [30] proposed the standard model of the mind, illustrated in Figure 3, that served us as a basis for creating the model of the athlete.

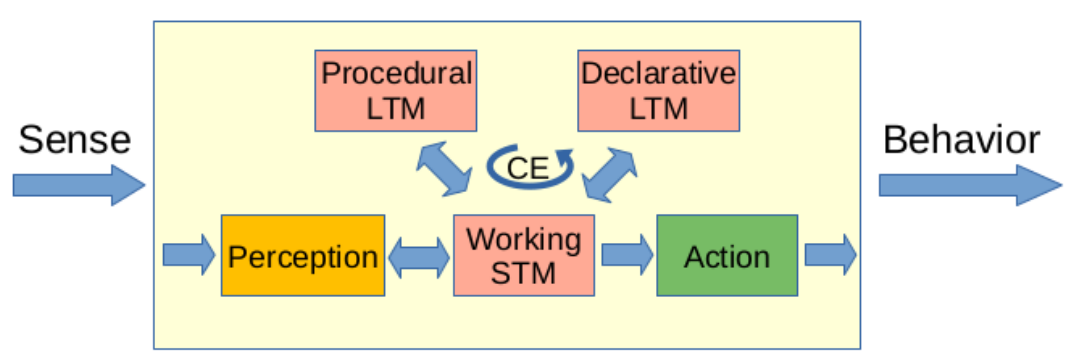

Figure 3. The standard model of the mind as proposed by Laird et al. [30].

According to the ACT-R model, humans perceive the real world using sensors in which signals are sensed from the environment. This information is transmitted into the brain. The sensors allow humans to see, hear, touch, smell and taste the world around them. The transmitted information is saved into specific sensory registers, e.g., iconic for sight, echoic for sound, haptic for touch, olfactory for smell, and gustatory for taste [28]. Attention filters the information, and creates a conscious picture of what the human "sees" by the perception component. The picture is processed in the working short-term memory (STM), referring to "a set of processes holding mental representations temporarily available for use in thought and actions" [31]. In this process, two kinds of long-term memories are involved, i.e., procedural and declarative. The former operates implicitly (unconsciousness), and refers to learned facts, like various motor skills (e.g., riding a bike), while the latter works explicitly (consciousness), and is devoted for remembering life events and facts. The last component represents the results of a cognition cycle, and, typically, refers to a selected motor action in which the human wishes to change the world, and, thus, determines their behavior.

The Central Executive (CE) serves for interactions between the mentioned components of the model. This plays the role of an operating system, characterized as a supervisory attentional system, which allows task switching and multiprocessing. 


\subsection{Digital Twin of an Athlete}

Obviously, the ACT-R model is too complex for implementing the athlete's DT in some individual sport disciplines, like cycling, running, and triathlon. Therefore, the simplified model, illustrated in Figure 4, can be used for these purposes.

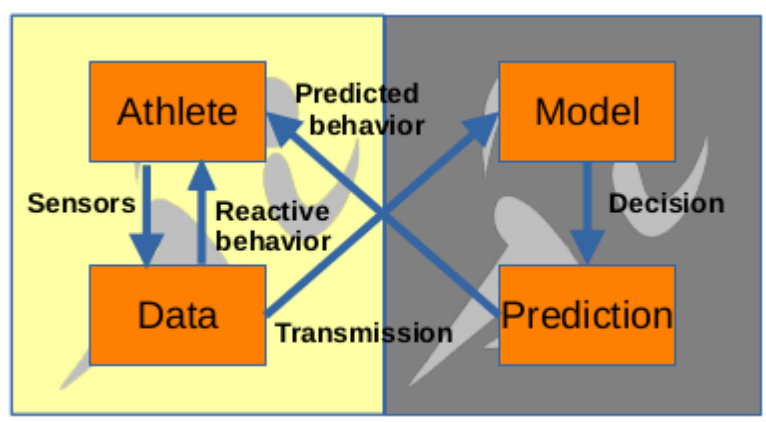

Figure 4. The proposed model of an athlete's DT.

As can be seen from this figure, the proposed model consists of:

- $\quad$ an athlete

- data

- model of the DT

- prediction

The athlete specifies the description of the real athlete in sport training with the corresponding context. The context includes the athlete's archive of performed training sessions, the whole training plan, his/her psycho-physical characteristics, as well as external factors which make an implementation of a particular sport training session more or less difficult. Data have arisen as a result of realizing the sport training session. They are accumulated through various sensors which monitor the quality of the performed session. Interestingly, these data are available to an athlete and they affect his/her reactive behavior. On the other hand, these are transmitted to the DT model capable of making-decisions related to the question of what to do in particular situation. Among many possible ML prediction methods, the most optimal reply to the situation is advised to the athlete by the DT as a predicted behavior. However, the athlete does not assume the advice unconditionally, but chooses between a reactive behavior created by he/she alone, and the predicted behavior obtained by his/her DT.

Indeed, the kind of ML prediction methods for the DT model are selected according to the demands of the sport disciplines. Production models can be used, when decisions are made using Association Rule Mining (ARM) in sport [32], while classical prediction models are implemented by connectionist models with Neural Networks (NN) [33] or Bayesian inference [34]. In our study, we focus on Recurrent Neural Networks (RNN) suitable for predicting the time series patterns [35].

\section{Illustrative Example}

The goal of our experimental work was to show that the DT model of an athlete can automatize the process of sport training substantially. In order to show that this concept could be used in real sport training, the DT was applied to sport training in cycling. The cycling was chosen intentionally because of the existing AST-control monitor that ensures the processor power for running the DT, as well as communication with the AST [22]. The following steps are necessary for adapting the proposed DT technology to the cycling:

- identification of cycling training specifics

- definition of the DT model in cycling

- integration of the DT model within the AST-control monitor

In the remainder of the paper, the mentioned steps are described in detail. 


\subsection{Specifics of a cycling training}

The power meter has brought a revolution in the process of cycling sport training. It is well known that preparing for a competition demands physical, as well as mental, changes of the athlete in the sport training. Indeed, the power meter takes care of making both changes simultaneously: On the one hand, the power meter ensures an athlete knows and increases his/her physical limitations, while, on the other hand, the athlete can prepare for the race mentally by knowing its demands, and, thus, performs sport training sessions that match these precisely.

Unfortunately, measuring intensity during a sport training session is difficult task. Typically, in practice, it is measured by the following measures [36]: Rating of Perceived Exertion (RPE), Heart Rate (HR), and PoWeR (PWR). Thus, the RPE estimates the intensity of the workout subjectively, because the athlete rates his/her perceived exertion in the numerical scale on a hunch. The HR is a more precise measure of effort, and measures how intensively an athlete is working, but the sport trainer does not gain any direct information about the effectiveness of the performed workout. The more accurate measure of intensity is PWR, which can be applied in cycling, and gauges the power that the cyclist places on the pedal directly as a product of torque by cadence [37].

The intensity of training load in cycling is prescribed by sport trainers using power zones. These present a simple training tool similar to the HR zones in running, where the training load is specified by HR intensity. The starting point for determining the personal power zones is the so-called Functional Threshold Power (FTP). This is unique for each cyclist, and indicates the maximum intensity that can be produced by the cyclist in one hour. In line with this, Coggan [37] proposed the so-called 1-hour test, where the road cyclist must overcome a 1-hour criterium at maximum intensity (i.e., power). If a shorter duration is considered, the measured FTP value needs to be decreased by an appropriate percent (e.g., $5 \%$ in the case of a 30-minute test).

The personal training zones are then constructed according to the hints illustrated in Table 1, from which it can be seen that there are seven power zones. These differ among each other regarding the percentage of the FTP, starting from less than $55 \%$ of the FTP by the active recovery PWR zone, to greater than $150 \$$ of FTP by the sprint power PWR zone.

Table 1. Power-based training zones according to Coggan [37].

\begin{tabular}{clc}
\hline Zone & PWR Zone Name & \% of FTP \\
\hline 1 & Active recovery & $<55$ \\
2 & Aerobic endurance & $56-75$ \\
3 & Tempo & $76-90$ \\
4 & Lactate threshold & $91-105$ \\
5 & $\mathrm{VO}_{2}$ max & $106-120$ \\
6 & Anaerobic capacity & $121-150$ \\
7 & Sprint power & $>150$ \\
\hline
\end{tabular}

\subsection{Digital Twin in Cycling}

The aim of the DT in cycling is to advise the real cyclist what to do during the realization of the training session in a particular situation. Indeed, it monitors the sensors incorporated into a wearable wireless device which measures the performances of the athlete and compares these achievements with those prescribed by the real trainer in the training plans. Based on the history of realized sport training sessions, the DT advises the athlete in which PWR intensity zones he/she must perform the current workout in order to satisfy the demands of the training plan. The proposed DT for cycling is based on the simplified cognitive model of a human (Figure 5), where signals from sensors are perceived by the perception component and transmitted to a "brain" component. 


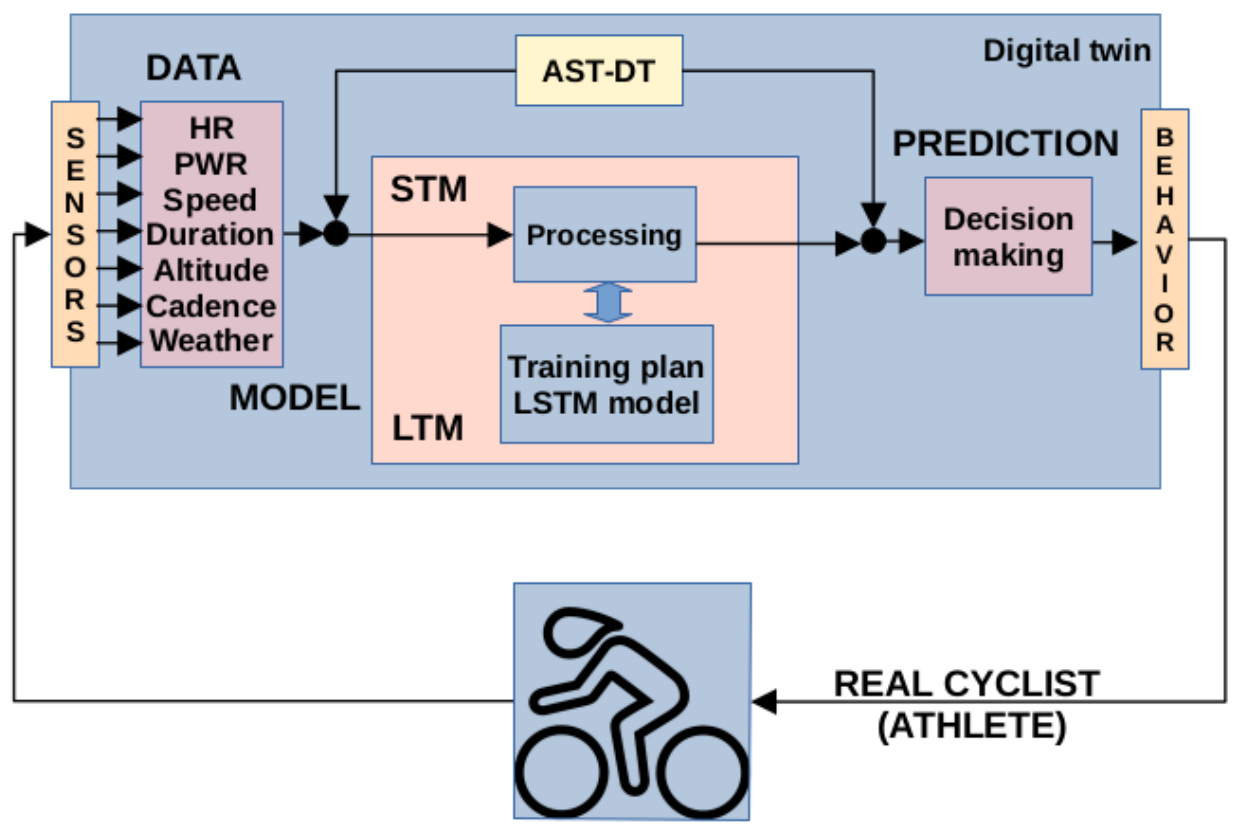

Figure 5. The proposed DT in cycling (The cyclist's icon was taken from [38]) which is licensed under Attribution 4.0 International (CC BY 4.0).

The component consists of STM and LTM, where the former is dedicated for processing information from the sensors, the latter to goal-oriented processing. The goal-oriented processing makes decisions according to the current training plan (the goal) and a cyclist's context obtained from the AST+. The cyclist's context is represented as a Long Short-Term Memory (LSTM) network, which incorporates a history of the cyclist's realized training activities, and it is suitable for predicting the cyclist's future behavior. The predicted behavior serves the cyclist as advice on how to react in a specific situation.

Typically, the other load indicators that can be measured during a training session besides the power meter, are as follows: heart rate, speed, duration, cadence, altitude, and weather. The HR is still valuable information of intensity load. Speed is a very interesting load indicator, but it is not the most accurate, due to its dependence on wind, weather, and altitude. The duration is an especially powerful load indicator for endurance cyclists, and has a direct relation with the calories' consumption, in the following sense: The longer the training, the larger the calories' consumption. The cadence load indicator measures the frequency of turning the wheel, and depends on the gear used for a specific situation, such as riding a straight road, climbing a hill, or sprinting. Thus, cycling trainers advise that the cyclists perform optimally when they ride the cycles in the lower gear with the higher cadence. Altitude allows the cyclist to monitor climbing the terrain during the sport training. This measure is obtained today using a Global Positioning System (GPS) that is part of the wearable monitoring device. The weather load indicator is part of the external factors that affect the performance of the realized sport training dramatically. Typically, the weather forecast consists of the following indicators: rain, sun, wind, humidity, and temperature.

As a DT model, the Recurrent Neural Networks (RNN) are considered that are appropriate for manipulating the sequential dependence among attributes in time-series datasets [35]. Indeed, the sport activities present a kind of time-series data. Unfortunately, the RNNs are sensitive on two problems, i.e., vanishing and exploding gradients, causing inherent instability of a back-propagation algorithm. The back-propagation algorithm works when gradients of the cost function with respect to the weights of the perceptrons consisting in the neural network are decreased by training with the input-output samples [35]. Thus, the vanishing of the gradient arises as a result of successive multiplication 
with a recurrent matrix of weights at various time-stamps, while exploding gradients mean that these blow up to large values by some combinations of input values.

The problems can be partially avoided by using the so-called Long Short-Term Memory (LSTM) networks controlling recurrence condition of how hidden states are propagated [39]. In line with this, these networks maintain the cell-state for each perceptron that represents a kind of long-term memory, where information is saved during arbitrary time intervals. Each cell is composed of three gates (i.e., input, forget, and output), which regulate the flow of information into and out of the cell. The LSTM networks are appropriate for deep learning.

In the DT for cycling, the archive of sport activities is presented as a set:

$$
\mathcal{A}=\left\{a_{1}, a_{2}, \ldots, a_{k}\right\},
$$

where elements $a_{i}$ for $i=1, \ldots, k$ denotes summaries of the realized sport activities, and $k$ determines the set size. Thus, the $i$-th element $a_{i}$, presenting the test input-output pair, is expressed as a tuple:

$$
a_{i}=\left\langle H R_{i}, P W R_{i}, v_{i}, t_{i}, \text { Alt }_{i}, \text { Cal }_{i}, \text { Weat }_{i}, \text { Class }_{i}\right\rangle,
$$

where

$$
\begin{aligned}
& H R_{i}=\text { heart rate, } \\
& P W R_{i}=\text { power, } \\
& v_{i}=\text { speed, } \\
& t_{i}=\text { time duration, } \\
& \text { Alt }_{i}=\text { altitude, } \\
& \text { Cad }_{i}=\text { cadence, } \\
& \text { Weat }_{i}=\text { weather conditions, } \\
& \text { Class }_{i}=\text { corresponding class }
\end{aligned}
$$

As a matter of fact, the Class ${ }_{i}$ attribute represents an output, while all the other attributes denote input values. The prediction using LSTM operates as follows: At first, an LSTM network is learned by supervision of the input/output pairs from an archive of sports activities. The learned network reflects the characteristics of the cyclist in training. Then, this LSTM network is applied for prediction, where the performance of partially realized training sessions is classified to the corresponding output class (i.e., the power zone), with regard to the input senses. Thus, the training session is prescribed by the AST+ in the following form:

$$
\mathcal{T}=\langle\text { power_zone,duration, frequency }\rangle,
$$

where power_zone indicates the intensity load of the training session, duration its endurance, and frequency determines how many times the particular training session needs to be repeated in a specific period of time.

Finally, the prediction is performed according to the specifications written in Algorithm 1, from which it can be seen that the algorithm, as input parameters, requires a training plan $\mathcal{T}$ and an archive of training sessions $\mathcal{A}$. The first step is LSTM training. A predicting process is performed inside the while statement that terminates when the implementation of the sport training session is complete. The process starts with sensing an environment, where the new training load indicators are perceived. Next, the averages of training load indicators are calculated using incremental averaging, in other words:

$$
\bar{x}_{t}=\bar{x}_{t-1}+\frac{x_{t}-\bar{x}_{t-1}}{t},
$$

where $\bar{x}_{t}$ denotes an average value of some training load indicator, and $x_{t}$ its value in a step $t$. Then, prediction with the LSTM network is conducted that suggests the suitable behavior to the athlete. Finally, the realized cycling training session is added to the archive. 


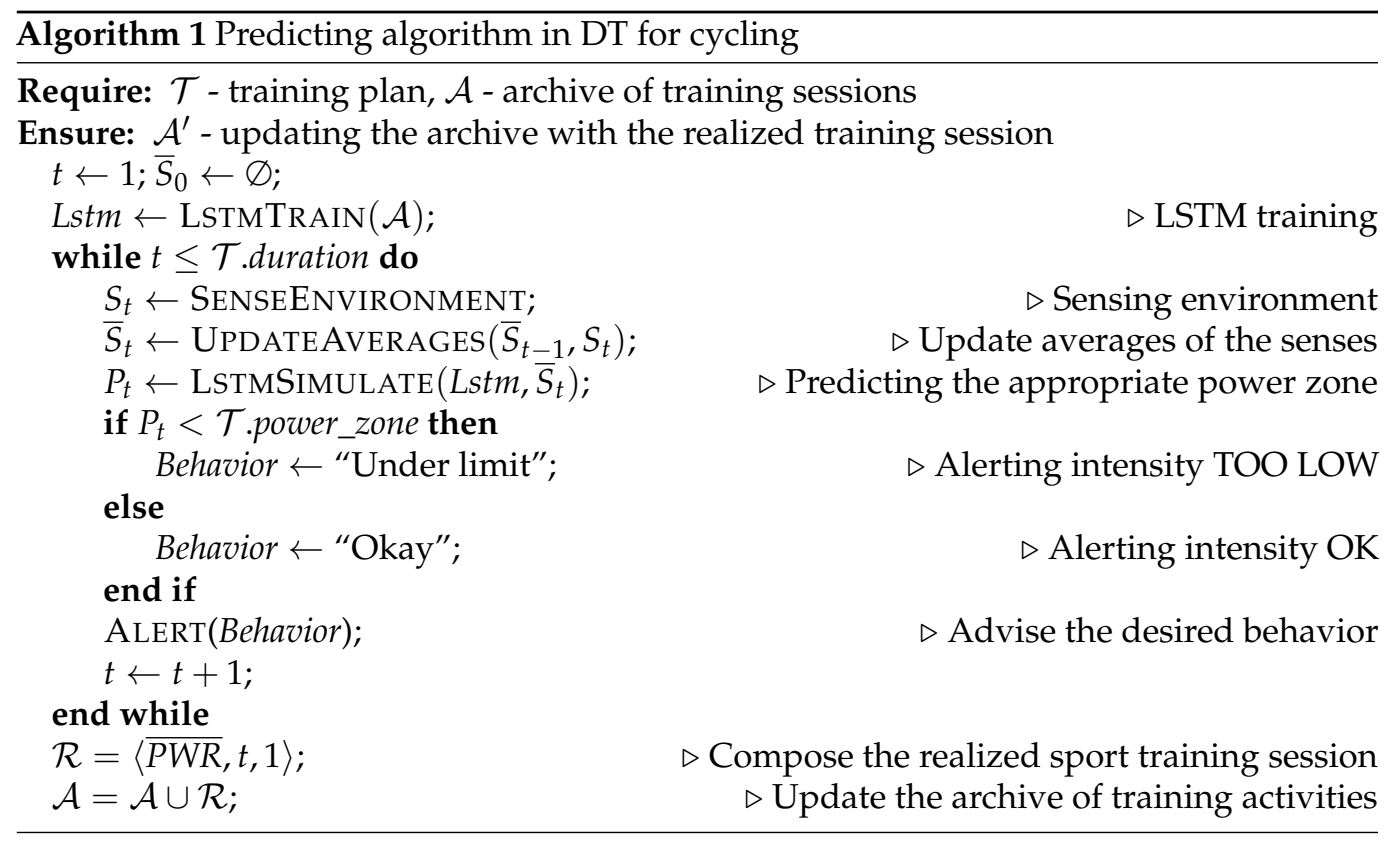

\subsection{Integration with the AST-Control Monitor}

Fister et al. [22] proposed a low cost embedded device for monitoring cyclist training sessions called the AST-control monitor. The device is totally flexible and open, as it supports connecting different sensors and is small enough to be embedded on the bicycle. Further, it supports the Internet connection, which allows the integration with the AST running on the host server computer as well as connections with other web services (like weather services for forecasting weather conditions). A simple Graphical User Interface (GUI) is developed on top, for interaction and feedback between the cyclist during individual training sessions.

The AST-control monitor is based on the Raspberry Pi computer, which has almost two advantages necessary for our study: (1) It can be mounted on the cycle, and (2) It supports a wide range of external sensors with which an environment can be sensed. The monitor is capable of carrying out the following functions:

- loading training plans from the AST+,

- monitoring the realization of the cycling training sessions,

- uploading the finished training activities to the AST+ for a detailed analysis.

The original AST-control monitor operates on the principle of feedback systems [40], and minimizes the difference between the prescribed and measured HR. On the other hand, the system is adapted for monitoring the interval cycling sessions. In line with this, three modifications need to be performed in order to satisfy our demands:

- a power meter must be connected to the Raspberry Pi via a USB ANT+ stick,

- the feedback system must be replaced with the proposed DT for cycling,

- the Graphical User Interface (GUI) must be adapted for monitoring the regular cycling training sessions instead of interval ones.

However, the purpose of the study was to find a cognitive predictive model of the athlete that could be run within the DT. Indeed, this DT could monitor the current results of the cyclist during the realization of a training session, and advise he/she what to do in specific situations in order to achieve the goal set by the AST+. Therefore, the testing of the AST-control monitor stays an unsolved task for the future. In the remainder of the paper, the advantages and weaknesses of the proposed solution are discussed in detail. 


\section{Discussion}

The technology of the DT for cycling, where the athlete's model (i.e., his/her DT) undergoes the same stresses as its real twin in overcoming the demands set by a cycling training session, is capable of advising the athlete to make the proper decisions in a specific situation. If the DT could simulate the behavior of the real human, it needs to imitate their kind of processing on a given work. Indeed, these kinds of problems are investigated by a computational cognitive science that is a part of cognitive psychology [21]. Actually, the task of cognitive psychology is the study of the internal processes involved in making sense of the environment and deciding on appropriate actions [21].

The goal of our study was to find a model of the DT whose behavior can be equivalent to the behavior of the real athlete in making decisions, with which its real counterpart is confronted. In line with this, we looked for an appropriate model in cognitive psychology. Here, decision-making is treated as a reactive behavior, where stimuli from the environment launch an appropriate response (i.e., behavior) by the human. Although behaviorism [41] (i.e., the dominant approach to psychology throughout the first half of the twentieth century) argued that reactive behaviour can arise without internal mental processes, the "cognitive revolution" overthrew this approach [42]. This means that the reactive behavior is not a simple mapping "stimuli $\rightarrow$ behavior", because each behavior demands some internal mental processing for making the proper decision. In the proposed cognitive model incorporated in DT for cycling, the decision-making process is implemented by an LSTM network.

The proposed cognitive model of the athlete has the following advantages: The model automates the process of realizing the sport training sessions. It mimics the mental processes of the human brain. As part of the DT, this model supplements the decisionmaking process of the athlete by advising him/her how to react in a specific situation. Thus, the connection between the digital and real parts of the DT is inherited. The decisionmaking process is based on an LSTM network that is capable of handling time-series data. Moreover, this process is context dependent (i.e., unique for each athlete in training). The athlete's context is held in the archive of realized sport training sessions and reflects the characteristics of the athlete. The concept allows monitoring more cyclists during the realization of sport training sessions simultaneously. Porting the cognitive model to the mobile device worn by cyclist during the training session. The theoretical model is universal (i.e., it can be used in various sport disciplines).

After integrating the DT for cycling to the AST-control monitor, some weaknesses were identified, which can be summarized as follows. The DT is deployed only to one sport discipline (i.e., cycling). The Raspberry Pi is impractical for use in other sports disciplines, like swimming and running, due to its size and weight. The mentioned device also has a problem with higher energy consumption. Moreover, the additional batteries increase the weight of the equipment that is crucial for cycling.

As follows from the discussion, the Raspberry Pi could present a bottleneck of the implemented solution. This means that a new platform must be found for running the proposed DT for cycling. The new platform might also be capable of porting the solution in other sport disciplines.

\section{Conclusions}

The AST that supplements the role of the real sport trainers covers all four phases of the sports training, i.e., planning, realization, control, and evaluation. Unfortunately, the realization and control phases have, typically, been conducted offline. However, the advances in computer science and telecommunication, especially, engaging the new ML methods and IoT sensors, allow for the monitoring of the realization and control phases online (i.e., automatically). Thus, the new ML methods enable new ways of analyzing the results of sport training sessions, while the new sensors allow monitoring these from a new point of view. For instance, the introduction of power meters has brought a revolution of sport training in cycling. 
In the sport training process, each athlete, especially in individual sports disciplines, is engaged as a unique individual, predestined by his/her mental and physiological characteristics. Therefore, the real sport trainers (as well as the new AST+) must take these into account by constructing the sport training process. Consequently, a model of an athlete needs to be found that is suitable for DT decision-making. The model is being searched for in cognitive computer science, where the cognitive scientists seek to understand how the mind works, i.e., to describe and predict human behavior [34]. In line with this, the simplified general cognitive model of human ACT-R is applied for modeling the athlete in sport training.

The DT is applied to cycling, where the wearable platform AST control monitor on the Raspberry Pi platform was already proposed by Fister et al. [22]. However, porting the theoretical DT of an athlete to the real DT in cycling that is based on the cognitive model of an athlete demands three steps: (1) identification of cycling specifics, (2) definition of the DT model in cycling, and (3) integration of the DT model with the AST control monitor. Indeed, the LSTM network is applied as a DT model in cycling. The results of the porting show the big potential of using the cognitive models in the process of sport training.

The presented study may be improved in several ways. These include: (1) application of the DT in other sport disciplines, (2) searching for a smaller wearable platform that could replace the rigid Raspberry Pi, and (3) testing the new technologies for DT models (e.g., Bayesian inference, graphical ML, etc.).

Author Contributions: Conceptualization, I.F.J., S.S.-S. and I.F.; methodology, I.F.J, A.I. and I.F.; software, I.F.J. and I.F.; validation, I.F.J., S.S.-S., A.G. and I.F.; data curation, I.F.J; writing-original draft preparation, I.F.J., S.S.-S., A.G., A.I., D.F. and I.F.; writing—review and editing, I.F.J., S.S.-S., A.G., A.I., D.F. and I.F.; visualization, D.F., I.F. All authors have read and agreed to the published version of the manuscript.

Funding: Iztok Fister Jr. is grateful for the financial support from the Slovenian Research Agency (Research Core Funding No. P2-0057). Iztok Fister is grateful for the financial support from the Slovenian Research Agency (Research Core Funding No. P2-0042-Digital twin). Dušan Fister is grateful for the financial support from the Slovenian Research Agency (Research Core Funding No. P5-0027). Akemi Galvez and Andres Iglesias have received funding from the project PDE-GIR of the European Union's Horizon 2020 research and innovation programme under the Marie SklodowskaCurie grant agreement no. 778035, and from the project TIN2017-89275-R funded by MCIN/AEI /10.13039/501100011033/FEDER “Una manera de hacer Europa”.

Conflicts of Interest: The authors declare no conflict of interest.

\section{References}

1. Chomsky, N.; Arnove, A. The Essential Chomsky; The New Press: New York, NY, USA, 2011.

2. Alpaydin, E. Introduction to Machine Learning; The MIT Press: Cambridge, MA, USA, 2014

3. Fister, I.J.; Yang, X.S.; Fister, I.; Brest, J.; Fister, D. A Brief Review of Nature-Inspired Algorithms for Optimization. arXiv 2013, arXiv:cs.NE/1307.4186.

4. Eberhart, R.C.; Shi, Y. Computational Intelligence: Concepts to Implementations; Morgan Kaufmann: Burlington, MA, USA, 2007.

5. Araújo, D.; Couceiro, M.S.; Seifert, L.; Sarmento, H.; Davids, K. Artificial Intelligence in Sport Performance Analysis; Routledge: London, UK, 2021.

6. Silacci, A.; Taiar, R.; Caon, M. Towards an AI-Based Tailored Training Planning for Road Cyclists: A Case Study. Appl. Sci. 2021, 11, 313. [CrossRef]

7. Rauter, S. New Approach for Planning the Mountain Bike Training with Virtual Coach. Trends Sport Sci. 2018, 2, 69-74.

8. Bezobrazov, S.; Sheleh, A.; Kislyuk, S.; Golovko, V.; Sachenko, A.; Komar, M.; Dorosh, V.; Turchenko, V. Artificial Intelligence for Sport Activitity Recognition. In Proceedings of the 2019 10th IEEE International Conference on Intelligent Data Acquisition and Advanced Computing Systems: Technology and Applications (IDAACS), Metz, France, 18-21 September 2019; Volume 2, pp. 628-632. [CrossRef]

9. Khasanshin, I. Application of an Artificial Neural Network to Automate the Measurement of Kinematic Characteristics of Punches in Boxing. Appl. Sci. 2021, 11, 1223. [CrossRef]

10. Chen, J.; Samuel, R.D.J.; Poovendran, P. LSTM with bio inspired algorithm for action recognition in sports videos. Image Vis. Comput. 2021, 112, 104214. [CrossRef] 
11. Sirico, F.; Romano, V.; Sacco, A.M.; Belviso, I.; Didonna, V.; Nurzynska, D.; Castaldo, C.; Palermi, S.; Sannino, G.; Della Valle, E.; et al. Effect of video observation and motor imagery on simple reaction time in cadet pilots. J. Funct. Morphol. Kinesiol. $2020,5,89$. [CrossRef] [PubMed]

12. Ardigò, L.P.; Palermi, S.; Padulo, J.; Dhahbi, W.; Russo, L.; Linetti, S.; Cular, D.; Tomljanovic, M. External Responsiveness of the SuperOpTM Device to Assess Recovery After Exercise: A Pilot Study. Front. Sport. Act. Living 2020, 2, 67. [CrossRef]

13. Sirico, F.; Palermi, S.; Massa, B.; Corrado, B. Tendinopathies of the Hip and Pelvis in Athletes: A Narrative Review. J. Human Sport Exercise 2020, 15, S748-S762.

14. Palermi, S.; Massa, B.; Vecchiato, M.; Mazza, F.; De Blasiis, P.; Romano, A.M.; Di Salvatore, M.G.; Della Valle, E.; Tarantino, D.; Ruosi, C.; et al. Indirect Structural Muscle Injuries of Lower Limb: Rehabilitation and Therapeutic Exercise. J. Funct. Morphol. Kinesiol. 2021, 6, 75. [CrossRef]

15. Fister, I.; Rauter, S.; Yang, X.S.; Ljubič, K.; Fister , I., Jr. Planning the sports training sessions with the bat algorithm. Neurocomputing 2015, 149, 993-1002. [CrossRef]

16. Fister, I.J.; Ljubič, K.; Suganthan, P.N.; Perc, M.; Fister, I. Computational intelligence in sports : Challenges and opportunities within a new research domain. Appl. Math. Comput. 2015, 262, 178-186. [CrossRef]

17. Fister, I.; Fister, I. Jr.; Fister, D. Computational Intelligence in Sports; Springer: Berlin/Heidelberg, Germany, 2019.

18. Bonidia, R.P.; Rodrigues, L.A.L.; Avila-Santos, A.P.; Sanches, D.S.; Brancher, J.D. Computational Intelligence in Sports: A Systematic Literature Review. Adv. -Hum.-Comput. Interact. 2018, 2018, 3426178. [CrossRef]

19. Farrokhi, A.; Farahbakhsh, R.; Rezazadeh, J.; Minerva, R. Application of Internet of Things and artificial intelligence for smart fitness: A survey. Comput. Netw. 2021, 189, 107859. [CrossRef]

20. Phatak, A.A.; Wieland, F.G.; Vempala, K.; Volkmar, F.; Memmert, D. Artificial Intelligence Based Body Sensor Network Framework-Narrative Review: Proposing an End-to-End Framework using Wearable Sensors, Real-Time Location Systems and Artificial Intelligence/Machine Learning Algorithms for Data Collection, Data Mining and Knowledge Discovery in Sports and Healthcare. Sport. Med.-Open 2021, 7, 79. [CrossRef]

21. Eysenck, M.W.; Keane, M.T. Cognitive Psychology: A Student's Handbook; Psychology Press, Routledge: New York, NY, USA, 2020.

22. Fister, I.J.; Fister, I.; Iglesias, A.; Galvez, A.; Deb, S.; Fister, D. On deploying the Artificial Sport Trainer into practice. In Proceedings of the 2021 8th International Conference on Soft Computing \& Machine Intelligence(ISCMI), IEEE Africa Council, Cairo, Egypt, 26-27 November 2021; in press.

23. Friel, J. The Cyclist's Training Bible: The World's Most Comprehensive Training Guide, 5nd ed.; VeloPress: Boulder, CO, USA, 2018.

24. Chen, K. Digital Twin; Royal Collins Publishing Company: Montreal, QC, Canada, 2021.

25. Clker.com, R.L. Light Grey Runner Clip Art. 2021. Available online: http://www.clker.com/clipart-light-grey-runner.html (accessed on 1 October 2021).

26. Shackelford, S.J. The Internet of Things: What Everyone Needs to Know; Oxford University Press: New York, NY, USA, 2020.

27. Russell, S.; Norvig, P. Artificial Intelligence: A Modern Approach, 3rd ed.; Prentice Hall: Englewood Cliffs, NJ, USA, 2010.

28. Hills, P.J.; Pake, M. Cognitive Psychology For Dummies; John Wiley \& Sons, Ltd.: Chichester, UK, 2016.

29. Rosenbloom, P.S.; Laird, J.E.; Lebiere, C. Précis of 'A Standard Model of the Mind'. In Proceedings of the Fifth Annual Conference on Advances in Cognitive Systems; Cognitive Systems Foundation: Troy, NY, USA, 2017.

30. Laird, J.E.; Lebiere, C.; Rosenbloom, P.S. A Standard Model of the Mind: Toward a Common Computational Framework across Artificial Intelligence, Cognitive Science, Neuroscience, and Robotics. AI Mag. 2017, 38, 13. [CrossRef]

31. Oberauer, K.; Lewandowsky, S.; Awh, E.; Brown, G.D.A. Benchmarks for models of short-term and working memory. Psychol. Bull. 2018, 144, 885-958. [CrossRef] [PubMed]

32. Fister, I. Jr.; Fister, I. Information Cartography in Association Rule Mining. IEEE Trans. Emerg. Top. Comput. Intell. 2021, 1-17. [CrossRef]

33. Han, J.; Kamber, M.; Pei, J. Data Mining: Concepts and Techniques, 3rd ed.; Morgan Kaufmann Publishers Inc.: San Francisco, CA, USA, 2011.

34. Farrell, S.; Lewandowsky, S. Computational Modeling of Cognition and Behavior; Cambridge University Press: Cambridge, UK, 2018. [CrossRef]

35. Aggarwal, C.C. Neural Networks and Deep Learning: A Textbook, 1st ed.; Springer Publishing Company, Incorporated: Berlin, Germany, 2018.

36. Clark, M.A.; Lucett, S.C.; Sutton, B.G. NASM Essentials of Personal Fitness Training, 4nd ed.; Jones \& Bartlett Learning: Burlington, MA, USA, 2014.

37. Allen, H.; Coggan, A.R.; McGregor, S. Training and Racing with a Power Meter, 3rd ed.; VeloPress: Boulder, CO, USA, 2019.

38. Icon-Icons Trial Bike Sport Cycling Icon. 2021. Available online: https://icon-icons.com/icon/trial-bike-sport-cycling/3985 (accessed on 1 October 2021).

39. Hochreiter, S.; Schmidhuber, J. Long Short-Term Memory. Neural Comput. 1997, 9, 1735-1780. [CrossRef] [PubMed]

40. Distefano, J.I.; Stubberud, A.R.; Williams, I.J. Schaum's Outline of Feedback and Control Systems, 3rd ed.; McGraw-Hill Education: New York, NY, USA, 2013.

41. Watson, J.B. Behaviorism; The People's Institute Publishing Co., Inc.: New York, NY, USA, 1924.

42. Tolman, E.C. Discussion: Interrrelationships between perception and personality: A symposium. J. Personal. 1949, 11, 48-50. [CrossRef] 